\title{
Mapping broadband single-photon wave packets into an atomic memory
}

\author{
J. Nunn, ${ }^{1, *}$ I. A. Walmsley, ${ }^{1}$ M. G. Raymer, ${ }^{2}$ K. Surmacz, ${ }^{1}$ F. C. Waldermann, ${ }^{1}$ Z. Wang, ${ }^{1}$ and D. Jaksch ${ }^{1}$ \\ ${ }^{1}$ Clarendon Laboratory, Oxford University, Parks Road, Oxford OX1 3PU, United Kingdom \\ ${ }^{2}$ Oregon Center for Optics and Department of Physics, University of Oregon, Eugene, Oregon 97403, USA
}

(Received 21 April 2006; published 22 January 2007)

\begin{abstract}
We analyze a quantum optical memory based on the off-resonant Raman interaction of a single broadband photon, copropagating with a classical control pulse, with an atomic ensemble. The conditions under which the memory can perform optimally are found, by means of a universal mode decomposition. This enables the memory efficiency to be specified in terms of a single parameter, and the control field pulse shape to be determined via a simple nonlinear scaling. We apply the same decomposition to determine the optimal configurations for read-out.
\end{abstract}

DOI: 10.1103/PhysRevA.75.011401

PACS number(s): 42.50.-p, 03.67.Dd, 03.67.Hk, 03.67.Lx

The conversion of optical information between photons and atoms forms the basis of a quantum interface that is a critical component of quantum communications networks and distributed quantum computers [1-3]. Such quantum information processing schemes require the ability both to move quantum information between nodes of a network, and to store the information. An important class of quantum optical memories is based on the interaction of individual photons with atomic ensembles, in which the information is transferred coherently from a single photon to a collective excitation of the atoms. If active feedback [4] is not used, these memories are generally optically dense, with the density controlled dynamically using an ancillary field [5-7].

In this paper we analyze a prototypical memory based on the off-resonant Raman interaction of a classical pulsed control field and a broadband signal photon in an atomic medium (see Fig. 1). The temporal structure of the signal photon is transferred by the control to a long-lived collective atomic excitation [5], or spin wave. This differs qualitatively from previous narrow band schemes [8], which demonstrated that quadrature squeezing could be transferred from optical fields to a collective atomic spin. We show how proper shaping of the control field allows the mapping of an input wave packet of arbitrary temporal shape to an output wave packet of a potentially different temporal shape. The dynamics are closely related to those investigated in proposals for entanglement generation via spontaneous [2,9] and, more recently, stimulated $[10,11]$ Stokes scattering. However the photon storage process is distinct from these, in that it exhibits an explicit time reversal symmetry; evinced by its fundamental mode structure. Spontaneous emission is suppressed as long as the excited state remains empty. Lossless unitary storage of broadband single photons - such as are commonly used in cryptographic and teleportation experiments $[1,12]$ — can therefore be implemented by detuning sufficiently from resonance [17]. Departure from resonance makes our scheme robust against inhomogeneities in the ensemble, so that solid state absorbers (e.g., semiconductor charge quantum dots [13]) could be substituted for the atoms. In addition, changing the detuning of the control pulse be-

\footnotetext{
*Electronic mail: j.nunn1@physics.ox.ac.uk
}

tween storage and retrieval allows for control over the frequency of the output state. In the following we consider propagation in the one-dimensional limit; a fully threedimensional model will be considered elsewhere.

The signal and control fields are Raman resonant, with center frequencies $\omega_{s}, \omega_{c}$, respectively. The classical control at time $t$ and position $z$ is represented by the Rabi frequency $\Omega(\tau)$, where $\tau \equiv t-z / c$ is the local time. The signal and spin wave amplitudes are described by the slowly varying annihilation operators $A(\tau, z)$, and $B(\tau, z)$, respectively [18]. The spin wave is a collective coherence of the form $B(\tau, z)$ $\propto \Sigma_{\beta}\left|1_{\beta}\right\rangle\left\langle 3_{\beta}\right| e^{-i\left(\omega_{s}-\omega_{c}\right) \tau}$, where the index $\beta$ runs over all atoms with position $z$ [10]. If the common detuning $\Delta$ of the signal and control pulses from single photon resonance is much larger than the signal bandwidth $\delta$, the control Rabi frequency $\Omega$, and the control bandwidth, the excited state $\left|m_{i}\right\rangle$ can be adiabatically eliminated. If the ensemble is prepared in the collective state $|0\rangle \equiv \otimes_{i}\left|1_{i}\right\rangle$, and if the population of the metastable state $\left|3_{i}\right\rangle$ is assumed to remain negligible, a linear theory can be used. The Maxwell-Bloch equations, in the slowly varying envelope approximation, are then found to be $[10,11]$

$$
\begin{gathered}
{\left[\partial_{\tau}-i|\Omega(\tau)|^{2} / \Gamma\right] B(\tau, z)=-\kappa^{*} \Omega^{*}(\tau) A(\tau, z) / \Gamma,} \\
{\left[\partial_{z}-i|\kappa|^{2} / \Gamma\right] A(\tau, z)=\kappa \Omega(\tau) B(\tau, z) / \Gamma,}
\end{gathered}
$$

where $\kappa$ is the signal field coupling and $\Gamma \equiv \Delta-i \gamma \equiv|\Gamma| e^{-i \theta}$ is the complex detuning, with real phase $\theta . \gamma$ arises from
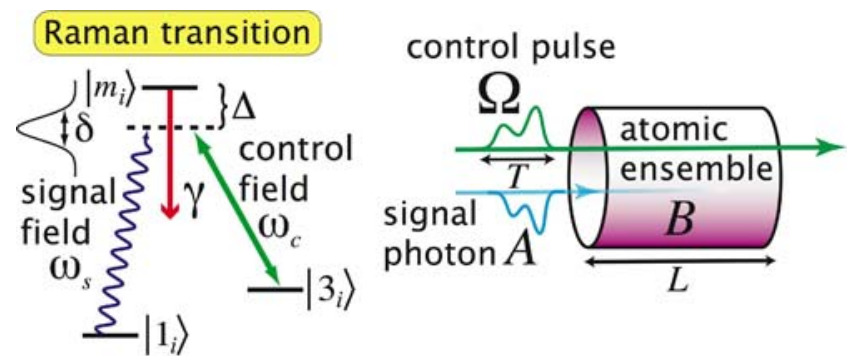

FIG. 1. (Color online) Left: the level structure of the $i$ th atom comprising a quantum memory for broadband photons, with bandwidth $\delta$. Right: a schematic of the read-in process for the quantum memory. 
dephasing processes, including spontaneous emission. We have not included the Langevin noise operator that formally accompanies these loss terms, since its contribution vanishes when normally ordered expectation values of $A$ and $B$ are taken. We neglect the slow decay of the spin wave over the short time scale of the memory interaction.

We now introduce a new set of scaled coordinates: the memory time $\epsilon(\tau) \equiv C \omega(\tau) / \omega(T)$, where $T$ is the duration of the interaction, and the effective distance $\zeta(z) \equiv C z / L$, with $L$ the length of the ensemble. Here $\omega(\tau) \equiv \int_{0}^{\tau}\left|\Omega\left(\tau^{\prime}\right)\right|^{2} d \tau^{\prime}$ is the integrated Rabi frequency and $C \equiv|\kappa| \sqrt{L \omega(T)} /|\Gamma|$ is a coupling parameter. We define dimensionless annihilation operators $\alpha(\epsilon, \zeta) \equiv \sqrt{\omega(T) / C} e^{-i \chi(\tau, z)} A(\tau, z) / \Omega(\tau)$ for the optical field (assuming $\kappa$ is real for simplicity) and $\beta(\epsilon, \zeta)$ $\equiv \sqrt{L / C} e^{-i \chi(\tau, z)} B(\tau, z)$ for the spin wave, where the exponent is $\chi(\tau, z) \equiv\left[\omega(\tau)+|\kappa|^{2} z\right] / \Gamma$. The first term in $\chi$ describes a Stark shift due to the control field; the second represents a modification of the signal group velocity. With these changes, the equations of motion reduce to the simple coupled system $\partial_{\zeta} \alpha=e^{i \theta} \beta ; \partial_{\epsilon} \beta=-e^{i \theta} \alpha$. The solution of these equations then holds for all control pulse shapes and arbitrary inputs. The coupling parameter $C$ sets the size of the region in $(\epsilon, \zeta)$-space over which the memory interaction is driven. For the case considered here, an atomic ensemble, $C$ can be rewritten in the form $C=\left(\pi \alpha_{f} \hbar / m_{e}\right)^{1 / 2} f \sqrt{N_{a} N_{c}} /$ $(|\Gamma| \mathcal{A})$, where $f$ is the geometric mean of the oscillator strengths for the signal and control transitions, $\mathcal{A}$ is the cross-sectional area of the control field, and $N_{a}\left(N_{c}\right)$ is the number of atoms (photons) interacting with (comprising) the control pulse. Here $\alpha_{f}$ is the fine structure constant, and $m_{e}$ is the electron mass.

The memory read-in and read-out must be unitary to function correctly. We now show that canonical evolution of the field operators $\alpha$ and $\beta$ is guaranteed by the classical structure of Eqs. (1) and (2) in the dispersive limit $\Delta \gg \gamma$. In this case, the phase $\theta$ vanishes and the following continuity relation is satisfied: $\partial_{\zeta} \alpha^{\dagger} \alpha+\partial_{\epsilon} \beta^{\dagger} \beta=0$. Integration of this expression over a square in $(\epsilon, \zeta)$-space yields the flux-excitation conservation condition

$$
N_{\alpha}(C)+N_{\beta}(C)=N_{\alpha}(0)+N_{\beta}(0),
$$

where the number operators $N_{\alpha}(\zeta) \equiv \int_{0}^{C} \alpha^{\dagger}(\epsilon, \zeta) \alpha(\epsilon, \zeta) d \epsilon$, and $N_{\beta}(\epsilon) \equiv \int_{0}^{C} \beta^{\dagger}(\epsilon, \zeta) \beta(\epsilon, \zeta) d \zeta$, count the number of signal photons at an effective distance $\zeta$, and the number of excitations of the spin wave at memory time $\epsilon$, respectively. Equation (3) must hold for arbitrary initial amplitudes $\alpha_{0}(\epsilon) \equiv \alpha(\epsilon, 0)$ and $\beta_{0}(\zeta) \equiv \beta(0, \zeta)$, which fixes the transformation $\left\{\alpha_{0}(\epsilon), \beta_{0}(\zeta)\right\} \rightarrow\left\{\alpha_{C}(\epsilon), \beta_{C}(\zeta)\right\}$ as unitary [where $\alpha_{C}(\epsilon)$ $\equiv \alpha(\epsilon, C)$ is the signal amplitude at the exit face of the ensemble, and $\beta_{C}(\zeta) \equiv \beta(C, \zeta)$ is the spin-wave amplitude at the end of the read-in process]. This allows the dynamics to be decomposed into a set of independent transformations between light-field and spin-wave modes [11,14]. In what follows we therefore concentrate on the dispersive limit, and consider the case $\Gamma \rightarrow \Delta ; \theta \rightarrow 0$. The solution of the dynamical equations is expressed by the scattering relations $[10,15]$

$$
\begin{aligned}
& \alpha_{C}(\epsilon)=\int_{0}^{C}\left[G_{1}(\epsilon-x, C) \alpha_{0}(x)+G_{0}(C-x, \epsilon) \beta_{0}(x)\right] d x, \\
& \beta_{C}(\zeta)=\int_{0}^{C}\left[G_{1}(\zeta-x, C) \beta_{0}(x)-G_{0}(C-x, \zeta) \alpha_{0}(x)\right] d x .
\end{aligned}
$$

The integral kernels are given by $G_{0}(p, q) \equiv J_{0}(2 \sqrt{p q})$, and $G_{1}(p, q) \equiv \delta(p)-\Theta(p) J_{1}(2 \sqrt{p q}) \sqrt{q / p}$, with the $n$th Bessel function of the first kind denoted by $J_{n}$, and where the Heaviside step function $\Theta$ ensures that the convolutions in Eqs. (4) and (5) respect causality.

The integral kernels $G_{0,1}$ - as they appear in Eqs. (4) and (5)-share symmetry under reflection about the line $C-x$ $=y$, where $y$ stands for the independent variable: either $\epsilon$ or $\zeta$. This symmetry, along with Eq. (3), allows us to decompose the kernels using input and output modes related by time reversal (or equivalently space reversal) as follows:

$$
\begin{aligned}
& G_{0}(C-\epsilon, \zeta)=\sum_{i=1}^{\infty} \phi_{i}(\zeta) \lambda_{i} \phi_{i}(C-\epsilon), \\
& G_{1}(\zeta-\epsilon, C)=\sum_{i=1}^{\infty} \phi_{i}(\zeta) \mu_{i} \phi_{i}(C-\epsilon),
\end{aligned}
$$

where $\left\{\phi_{i}\right\}$ is a complete orthonormal set of real mode functions and where the real, positive singular values satisfy the constraint $\lambda_{i}^{2}+\mu_{i}^{2}=1, \forall_{i}$.

The ensemble begins the read-in process in the state $|0\rangle$, and we are free to replace $\beta_{0}(\zeta)$ by its expectation value; $\beta_{0}(\zeta) \rightarrow 0$. With the above decomposition, Eq. (5) then describes a mapping of the optical input mode $\phi_{i}(C-\epsilon)$ to the output spin-wave mode $\phi_{i}(\zeta)$, with transfer amplitude $-\lambda_{i}$, for each $i$. Transforming from memory time $\epsilon$ back to local time $\tau$, the normalized input modes are written $\Phi_{i}(\tau) \equiv \sqrt{C / \omega(T)} e^{i \chi(\tau, 0)} \Omega(\tau) \phi_{i}[C-\epsilon(\tau)]$. The read-in efficiency can be quantified by evaluating the expectation value of the spin-wave number operator $\left\langle N_{\beta}(C)\right\rangle$ at the end of the read-in process. Expanding an incident signal wave packet $\xi(\tau)$ using the $\Phi_{i}$, the read-in efficiency is expressed as $\left\langle N_{\beta}(C)\right\rangle=\Sigma_{i} \lambda_{i}^{2}\left|\xi_{i}\right|^{2}$, with the $i$ th overlap given by $\xi_{i}$ $\equiv \int_{0}^{T} \xi^{*}(\tau) \Phi_{i}(\tau) d \tau$. When $\left\langle N_{\beta}(C)\right\rangle=1$, the read-in works perfectly. In Fig. 2 the first five transfer amplitudes are plotted as a function of $C$. These are found using the eigenvalue equation

$$
\int_{0}^{C} J_{0}(2 \sqrt{x y}) \phi_{i}(y) d y=\lambda_{i} \phi_{i}(x)
$$

which we solve numerically using a $500 \times 500$ square grid. It is desirable to limit the energy of the control pulse, so we should find the minimum coupling parameter $C$, which permits complete storage of the signal. For $C \approx 2$ the lowest mode achieves its optimal efficiency $\lambda_{1} \approx 1$, but higher modes remain poorly coupled. The efficiency of the memory is therefore maximized by setting $\xi_{1}=1 ; \quad \xi_{i \neq 1}=0$, so that 


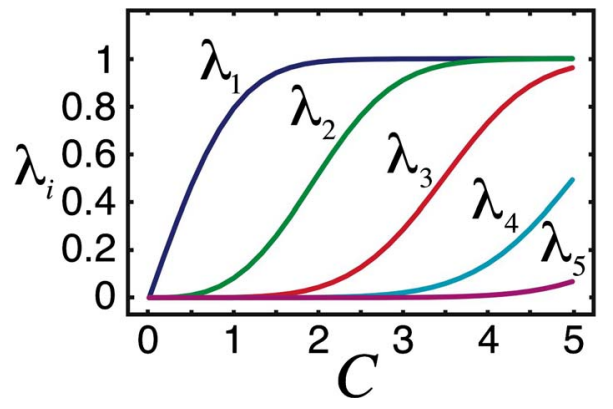

FIG. 2. (Color online) The five largest singular values of the kernel $G_{0}$, plotted as a function of the coupling parameter $C$.

$\left\langle N_{\beta}(C)\right\rangle=\lambda_{1}^{2} \approx 1$ (for $C \geqslant 2$ ). To do this, it is necessary to shape the control field so that $\Phi_{1}(\tau)=\xi(\tau)$. If Gaussian optics $\left(\mathcal{A} \sim c L / \omega_{s}\right)$ are used to illuminate a region a few $\mathrm{cm}$ long in a typical atomic vapor $(f \sim 1)$ of modest density $\left(\sim 10^{20} \mathrm{~m}^{-3}\right)$, with $100 \mathrm{~nJ}$ control pulses, a 1 ps photon wave packet can be stored optimally, with $C=2$. In practice mode matching could be achieved through measurement and feedback: the signal and control field sources are locked to a phase reference and operated in pulsed mode. The control pulse profile is characterized [16], and augmented until the transmission of the signal is minimized. Figure 3 shows the result of a simple optimization to find the control pulse shape which mode matches the lowest input mode to a Gaussian signal photon, for $C=2$. The photon is absorbed with $\left\langle N_{\beta}(C)\right\rangle \approx 0.96$; the small transmission probability is due to the limitations of our numerical mode matching optimization.

Once a properly mode matched photon has been read into the quantum memory, the ensemble is left in the output mode $\phi_{1}(\zeta)$, with probability amplitude $-\lambda_{1}$. We now consider the effect of sending a second control pulse, propagating in the same direction as the initial control pulse, into the ensemble. The center frequency, bandwidth, and intensity of this readout pulse may differ from that of the first control pulse (herein the read-in pulse). Let us use a superscript $r$ to indicate those quantities associated with the read-out. We neglect decoherence and dephasing of the spin wave over the storage period and set $B^{r}(0, z)=B(T, z)$. This provides us with one boundary condition; the second is that the signal field begins in its vacuum state at the start of the read-out process,
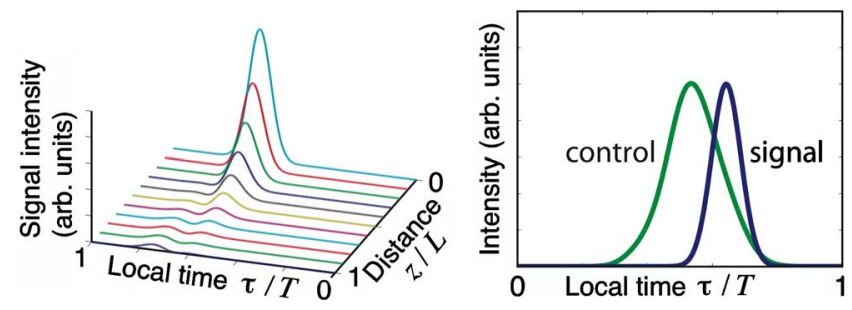

FIG. 3. (Color online) Left: the intensity $\left\langle A^{\dagger}(\tau, z) A(\tau, z)\right\rangle$ of a Gaussian signal photon $\left|1_{\xi}\right\rangle$, with wave packet amplitude $\xi(\tau)$ $\propto \exp \left\{-2 \ln 2\left[\left(\tau-\tau_{0}\right) / \sigma\right]^{2}\right\}$, as it propagates through an atomic ensemble with $C=2$. Here $\sigma=T / 8, \tau_{0}=2 T / 3$. Right: the optimized control field intensity is shown along side the initial signal field intensity (scaled for clarity).

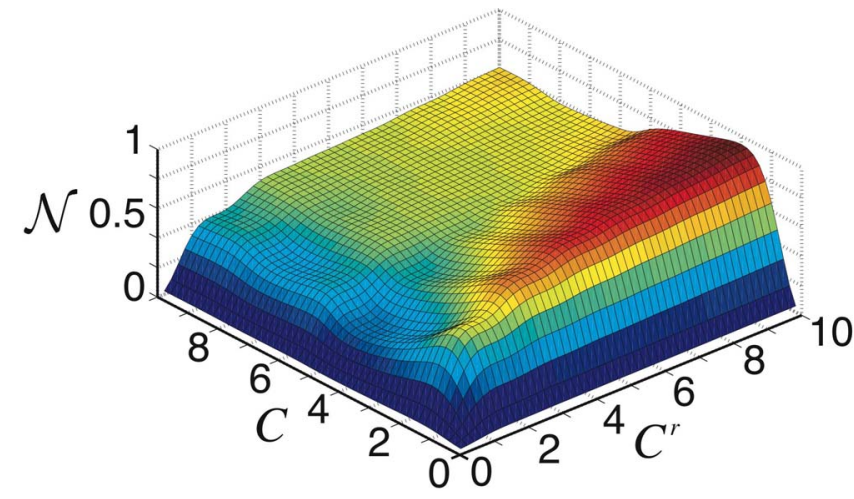

FIG. 4. (Color online) The photon retrieval probability $\mathcal{N}$, for forward read-out, plotted as a function of the read-in and read-out coupling parameters $C$ and $C^{r}$.

$\left\langle N_{\alpha}^{r}(0)\right\rangle=0$. The efficiency of the read-out depends upon the degree to which the spin-wave mode $\psi_{1}(z)$ $\equiv \sqrt{C / L} e^{i \chi(T, z)} \phi_{1}(C z / L)$ (written in terms of the ordinary spatial variable $z$ ) overlaps with the input modes $\Psi_{i}^{r}(z)$ $\equiv \sqrt{C^{r} / L} e^{i \chi^{r}(0, z)} \phi_{i}^{r}\left[C^{r}(1-z / L)\right]$ for the read-out process. The functions $\left\{\phi_{i}^{r}\right\}$ solve the eigenvalue equation (8), with $C$ replaced by $C^{r}$. A measure of the efficiency of the memory is the expectation value of the output photon number operator $\mathcal{N} \equiv\left\langle N_{\alpha}^{r}\left(C^{r}\right)\right\rangle=\lambda_{1}^{2} \Sigma_{i} \lambda_{i}^{r 2}\left|f_{i}\right|^{2}$, with the read-out overlaps defined by $f_{i} \equiv \int_{0}^{L} \psi_{1}^{*}(z) \Psi_{i}^{r}(z) d z$. The parameter $\mathcal{N}$ is the probability of retrieving a photon from the ensemble at read-out, given that a single mode matched photon was sent in with the read-in pulse. If the detuning does not change too much, so that $\left|\Delta^{r}-\Delta\right| / \Delta \ll \Delta^{r} /\left(|\kappa|^{2} L\right) \sim \sqrt{N_{c}^{r} / N_{a}}$, then the phases $\chi$, $\chi^{r}$ of the spin-wave modes approximately cancel, and then the stored spin wave is phase matched to the read-out modes. In Fig. 4 the variation of $\mathcal{N}$, under this approximation, is plotted as a function of the read-in and read-out coupling parameters $C$ and $C^{r}$. If $C=C^{r}$, then the lowest read-out mode is just the mirror image of the spin-wave mode. For small $C$, the spin-wave mode is monotonic, and relatively flat; $f_{1}$ is therefore large. However the transfer amplitudes $\lambda_{1}$ and $\lambda_{1}^{r}$ remain small, so the retrieval probability is low. Increasing $\lambda_{1}$ requires a larger $C$, but this produces a more asymmetric spin-wave mode, and $f_{1}$ falls. It is then necessary to increase $C^{r}$ above $C$, so that higher modes, with which the spin wave mode overlaps significantly, are efficiently coupled to the optical field. The retrieval probability is maximized along the line $C \approx 2$, which represents the optimal coupling for the read-in process. However, a read-out coupling parameter in excess of 10 is required to achieve $\mathcal{N}$ $\geqslant 0.95$. Note also that mode matching to the lowest mode at read-in is the best strategy for maximizing the memory efficiency. Mode matching to a higher mode, or some combination of modes, simply increases the optimal read-in coupling above $C=2[19]$.

The time reversal symmetry between the input and output modes makes the read-out for this scheme a nontrivial problem. Simply repeating the read-in process (so that $C=C^{r}$ ) results in poor performance of the memory. However, the dramatic increase in coupling strength required to extract the stored excitation fully, may make a naïve increase in control 
pulse energy at read-out prohibitively difficult to realize. An alternative method to boost the coupling is to reduce the bandwidth of the read-out pulse, along with its detuning $\Delta^{r}$. The photon recovered from the memory in this way would be frequency shifted (according to the Raman resonance condition), and temporally stretched (since its bandwidth would be diminished as well). Such a memory would act as a photon transducer, storing broadband photons and converting them to narrow band photons with tunable frequency on demand.

Note that switching the propagation direction of the readout pulse sends $\phi_{i}(C z / L) \rightarrow \phi_{i}[C(1-z / L)]$, so that the spinwave mode overlaps exactly with the lowest read-out mode with $C^{r}=C$, and we should obtain $\mathcal{N}=\lambda_{1}^{4}$. Unfortunately the read-out process is no longer phase matched in this situation, and the overlap integrals $f_{i}$ vanish. However, a solid-state implementation might allow this kind of reverse read-out with the use of quasi-phase-matching, in which the sign of the read-out coupling parameter $C^{r}$ is periodically flipped along the length of the ensemble.

The phase matching problem is obviated in the limit of vanishing Stokes shift, but then the ground state $|0\rangle$ must be prepared artificially with high purity: if the state $\left|3_{i}\right\rangle$ is initially populated, or if selection rules allow residual coupling of the control to the ground state, the memory fidelity at the level of single quanta is greatly reduced. Furthermore, if signal and control are spectrally indistinguishable, another degree of freedom should be used to differentiate between them. Typically, polarization affords discrimination to a part in $\sim 10^{6}$, but then the control should not contain more than a million photons. A large Stokes shift is therefore desirable, and correct phase matching at read-out is crucial for efficient retrieval. The above considerations demonstrate the importance of propagation effects in the design of an optical quantum memory.

We have shown that the off-resonant Raman configuration for a $\Lambda$-type ensemble quantum memory can be used to implement deterministic, controllable, and unitary transfer of the temporal structure of broadband single photons to a stationary spin wave, in the adiabatic regime. The dynamics are understood and optimized using a universal mode decomposition, valid for all control pulses and arbitrary input states. The modes are computationally simple to evaluate, and only a few of them are required to approximate the interaction faithfully. The optimal fidelity of the memory depends only upon a single dimensionless parameter $(C)$, which defines an equivalence class for memories with different physical implementations.

The authors gratefully acknowledge the support of Hewlett-Packard and the EPSRC (UK) through the QIP IRC (GR/S82176/01). One of the authors (M.G.R.) thanks the QIP IRC for support during his stay in Oxford, and also acknowledges the US NSF (AMOP) for support. The research of one of the authors (D.J.) was supported in part by The Perimeter Institute for Theoretical Physics. One of the authors (I.A.W.) was supported in part by the European Commission under the Integrated Project Qubit Applications (QAP) funded by the IST directorate as Contract No. 015848. One of the authors (J.N.) thanks A. V. Gorshkov for clarifying numerous points.
[1] Nicolas Gisin, Grigoire Ribordy, Wolfgang Tittel, and Hugo Zbinden, e-print, quant-ph/0101098.

[2] L.-M. Duan, M. D. Lukin, J. I. Cirac, and P. Zoller, Nature (London) 414, 413 (2001).

[3] H.-J. Briegel, W. Dür, J. I. Cirac, and P. Zoller, Phys. Rev. Lett. 81, 5932-5935 (1998).

[4] Jacob Sherson, Brian Julsgaard, and E. S. Polzik, e-print, quant-ph/0601186.

[5] M. Fleischhauer and M. D. Lukin, Bull. Am. Phys. Soc. 65, 022814 (2002).

[6] M. D. Lukin, Rev. Mod. Phys. 75, 457 (2002).

[7] B. Kraus, W. Tittel, N. Gisin, M. Nilsson, S. Kroll, and J. Cirac, Phys. Rev. A 73, 020302 (2006).

[8] A. E. Kozhekin, K. Mølmer, and E. Polzik, Phys. Rev. A 62, 033809 (2000).

[9] L.-M. Duan, J. I. Cirac, and P. Zoller, Phys. Rev. A 66, 023818 (2002).

[10] M. G. Raymer, J. Mod. Opt. 51, 1739 (2004).

[11] Wojciech Wasilewski and M. G. Raymer, Phys. Rev. A 73,
063816 (2006).

[12] Rupert Ursin, Thomas Jennewein, Markus Aspelmeyer, Rainer Kaltenbaek, Michael Lindenthal, Philip Walther, and Anton Zeilinger, Nature (London) 430, 849 (2004).

[13] Wang Yao, Ren-Bao Liu, and L. J. Sham, Phys. Rev. Lett. 95, 030504 (2005).

[14] Samuel L. Braunstein, e-print, quant-ph/9904002.

[15] M. G. Raymer and J. Mostowski, Phys. Rev. A 24, 1980 (1981).

[16] C. Iaconis and I. A. Walmsley, Opt. Lett. 23, 792 (1998).

[17] Shortly after our work was completed, Gorshkov et al. presented a paper, e-print, quant-ph/0604037, claiming that the storage of broadband photons on resonance is possible given sufficient atomic density and unlimited control pulse energy.

[18] We work in the linear regime where the operator nature of the variables is not manifest; we retain the operator notation to emphasize that our results hold for nonclassical input states.

[19] This point will be clarified in a forthcoming paper. 